\title{
Prediction Based Energy Balancing Forwarding in Cellular Networks
}

\author{
Jian-Jun YANG, Ju SHEN \\ Department of Computer Science and Information Systems, University of North Georgia, Oakwood, GA, USA \\ Department of Computer Science, University of Dayton, Dayton, OH, USA \\ e-mail: jianjun.yang@ung.edu, jshen1@udayton.edu
}

\begin{abstract}
In the recent cellular network technologies, relay stations extend cell coverage and enhance signal strength for mobile users. However, busy traffic makes the relay stations in hot area run out of energy quickly. Energy is a very important factor in the forwarding of cellular network since mobile users(cell phones) in hot cells often suffer from low throughput due to energy lack problems. In many situations, the energy lack problems take place because the energy loading is not balanced. In this paper, we present a prediction based forwarding algorithm to let a mobile node dynamically select the next relay station with highest potential energy capacity to resume communication. Key to this strategy is that a relay station only maintains three past status, and then it is able to predict the potential energy capacity. Then, the node selects the next hop with potential maximal energy. Moreover, a location based algorithm is developed to let the mobile node figure out the target region in order to avoid flooding. Simulations demonstrate that our approach significantly increase the aggregate throughput and decrease the delay in cellular network environment.
\end{abstract}

\section{Introduction}

The new technology of Cellular Networks are able to make a lot of mobile users(cell phones) connect a relay station near to them and then they connect to a base station. This technology enhances the service of a single relay station. However, the energy costs of relay stations go up very fast. Hence, the efficiency of energy utilization in Cellular Networks has attracted great interest in academic research and industrial applications, such as 3GPP and ITU[1]. Consequently, a new area called green cellular networks appeared. The European Commission is working on many new projects to taking care of the energy issue of Cellular Network systems. There systems cover many research topics, such as energy aware radios, energy efficient network design, cognitive radio for power saving, cooperative schemes[2]. The study of Energy in Cellular Network is in all layers of the protocols and a wide variety of system architectures.

The busy traffic near a relay station often makes the energy of the relay station go down very quickly. However, other relay stations with low traffic are idle with high energy. Many cell phones are connected in a hot relay station because by a kind of forwarding algorithm, such as shortest path forwarding, a large number of cell phones are connected to the same relay station. But, it is important to find the best tradeoff between energy efficiency and the performance [3]. In order to balance the energy utilization among different cells, it is needed to transfer the overloaded traffic from hot cells to neighboring cooler ones. The challenge is how to balance the energy utilization of the relay stations in order to get the best tradeoff among all the relay stations in a cellular network.

Scholars have come up with many research topics in terms of energy efficiency with network performance[1][4], such as balancing deployment cost and throughput, balancing achievable rate, balancing the bandwidth utilized, and balancing average end-to-end service delay. In this paper, we propose a prediction based algorithm for a mobile node(cell phone) to find one of its neighboring relay stations with possibly maximal energy in the next time, and then its chooses that relay station instead of using the fixed relay station to forward data. A relay station only saves its last three energy status, and then it predicts the potential energy in the next time. We further devise an algorithm to let the mobile node narrow the region on finding its neighboring relay stations in order to avoid opposite forwarding.

The rest of the paper is organized as follows. Section II discusses the related research on this topic. Section III proposes a novel method that select the best relay station. We evaluate the proposed schemes by simulations and describe the performance results in Section IV. Section V concludes the paper. 


\section{Related Work}

Scholars have developed many approaches to solve energy utilization questions in Cellular Networks. The approaches are classified into several categories. The first one is based on dynamic channel allocation, meaning channel borrowing from cooler cell[5][9]. The second one is based on Base Station selection [6]. The third type contains strategies based on power control and cell breathing [6][7]. The last category consists of strategies on relay-assisted traffic transfer [8].

The research of multi-hop wireless network is basic yet very important in wireless networks. Many scholars also work on energy efficiency topics with the infrastructure of multi-hop wireless networks, meaning multi-hop cellular network[15][16]. In this kind of structure, relay stations are network components that are used to store and forward data received from base stations to mobile users(cell phones), and vice versa. Deploying relay stations can extend the coverage and enhance the signal strength, which help improve the performance for mobile users near the edge of the cell. In addition, the appearance of multi-hop cellular network transfers over-loaded traffic from hot cells to cooler cells by relay stations. The relay-based load balancing schemes are flexible and introduce less interference.

A scheme to separate the networks to two parts were designed in[10], which divided the channels into two parts, one for the ad-hoc overlay network, the other for the cellular network. In[9][11], the authors proposed dynamic load balancing schemes in the integrated cellular and ad-hoc relaying systems. The ad-hoc relaying stations include an overlay ad-hoc network, which helps relay traffic among different cells. A dynamic traffic-aware base station switching mode was designed in [12], where the base station can change its operating modes between standard base station operations and switching to relay station mode, the so called BS-RS Switching model. Moreover, the propagation distance was shortened in this approach [10].

\section{Problem Formulation}

\subsection{The basic idea}

In cellular networks, each base station covers a number of cell phones in a hexagonal cell. We consider a cellular network containing a set of base stations(BS), relay stations(RS) and mobile users(cell phones)(MU), denoted by $\{\mathrm{A} 1, \mathrm{~A} 2, \mathrm{~A} 3 \ldots\},\{\mathrm{S} 1, \mathrm{~S} 2, \mathrm{~S} 3 \ldots\}$, and $\{\mathrm{c} 1, \mathrm{c} 2, \mathrm{c} 3 \ldots$,$\} . Each$ $\mathrm{BS}$ is located at the center of the cell. Each RS is located at a boundary of cells. The traditional communication between two mobile users ci to cj is that ci communicates to its base station $\mathrm{Ai}$, then $\mathrm{Ai}$ communicates to $\mathrm{Aj}$ which covers $\mathrm{cj}$, and then $\mathrm{Aj}$ communicates to $\mathrm{cj}$. In new generation cellular network, the relay stations are used to assist communications between cell phone to cell phone or cell phone to base station. Fig. 1 shows an instance with several base stations, relay stations and cell phones, where the lower right cell is a hot cell with a large number of cell phones and busy data traffic and the other cells are cooler cells with less cell phones. Note that the cell phones in all figures of this paper represent active cell phones.

Data transmission takes place when a cell phone(initiator) intends to talk to another cellphone(receiver). The scenario is simple if the two are in the same cell[15]. We study the circumstance that the receiver is in another cell. In Fig. 1, the communication between $\mathrm{c}_{1}$ and $\mathrm{c}_{2}$ is such an instance, in which data is relayed from $c_{1}$ to $S_{1}$, then from $S_{1}$ to $A_{1}$, and continues until

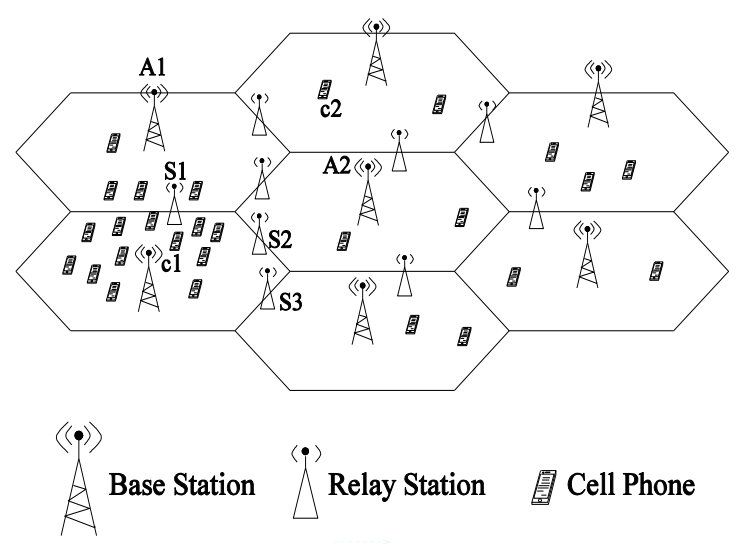

Figure 1. Topology of a Cellular Network

it reaches $c_{2}$. In the scenario, $S_{1}$ is hot with a lot of cell phones nearby. Hence, its energy is going down very quickly. However, $\mathrm{S}_{2}$ covers less cell phones and its energy is going down slowly. Therefore, our focus in this paper is to design an approach to help $c_{1}$ find $S_{2}$ or even $S_{3}$ as the next relay station to forward the message, and to bypass the hot relay station $\mathrm{S}_{1}$ as well.

Since a mobile cell phone is able to select a relay station and hand over to it[15], we develop an approach to let each mobile node find the RS with highest potential energy and hand over to it. We start our basic idea by a simplified case as Fig. 2. Suppose c1 intends to talk to $\mathrm{c} 2$. If $\mathrm{c} 1$ selects $\mathrm{S} 1$ to conduct relay, it may not be a good choice because S1 is busy and may run out of energy shortly. Our goal is to let c1 select the relay station that will have the highest potential energy. In our approach, each RS can predict its future energy right after this time slot based on its historical and current energy, which is defined as potential energy of the $\mathrm{RS}$ in this paper. In this example, our strategy will make c1 choose S2.

\subsection{Computation of Potential Energy}

The mobile initiator needs to know which relay station is the one that has the potential highest energy. The energy varying trend is essential to determine an RS's potential energy. Suppose the current time is k. A relay station $\mathrm{Si}$ saves its Energy changes of the last three times t0, t1, and t2. By Computational Method, we define a factor $L_{i, k}$ as follows: 


$$
L_{i, k}=\prod_{\substack{i=0 \\ i \neq k}}^{m} \frac{\left(t-t_{i}\right)}{\left(t_{k}-t_{i}\right)} E_{i, m}
$$

Where $E_{i, m}$ is the energy of $\mathrm{RS}_{\mathrm{i}}$ at time $\mathrm{m}$.

The for a near future time $\mathrm{p}$, the energy of $\mathrm{RS}_{\mathrm{i}}$ can be calculated as:

$$
E_{i, p}=\sum_{k=0}^{p} L_{i, k}
$$

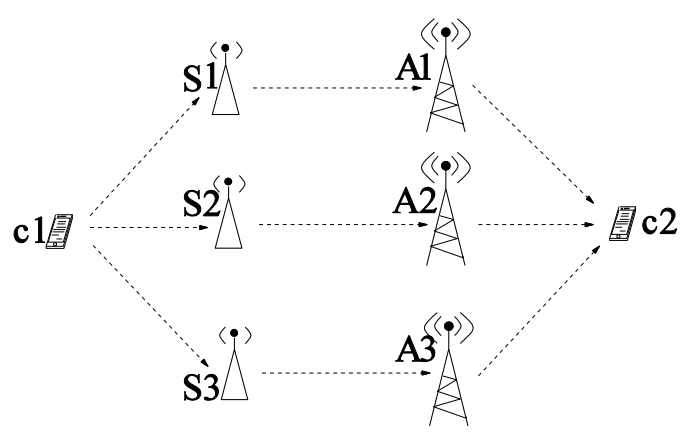

Figure 2. The communication initiator making a chocie of a relay station

Each RS Si keeps its energy status of the last three times. When a mobile user ci intends to send packages to another mobile user cj, ci sends a call for energy message to its neighbor relay stations. Then it figures out the RS with highest energy, and then selects the RS as relay station. Obvious, ci will not find an RS in the opposite direction of cicj as the relay station. In cellular networks, each device is equipped with GPS and hence it knows its location. We assume the initiator knows the target's location. It is very common in geographic routing [13][14]. When ci intends to set up a talk with cj, cicj is virtually connected, then cicj is used as the internal bisector to make an angle of 120 degrees. Then ci finds its relay station within this angle.

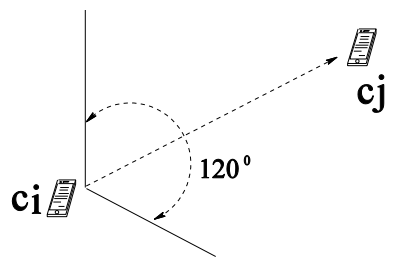

Figure 3. The communication initiator figureing out the region of next relay station

\section{$4 \quad$ Algorithm}

Algorithm 1 Energy data request
1. A mobile user $\mathrm{c}_{\mathrm{i}}$ figures out the 120 degree area.

2. $\mathrm{C}_{\mathrm{i}}$ send energy data request message to its neighboring relay station within the 120 degree area.

In algorithm 1, when a mobile node ci intends to set up a talk with another mobile node cj, ci figures out the 120 degree area with its location and the location of cj. Then ci sends an energy data request message to its neighbor relay station within the area.

Algorithm 2 Prediction for future energy

1. A relay station $S_{i}$ keeps its energy of the last three times.

2. $\mathrm{S}_{\mathrm{i}}$ calculates $L_{i, k}$ with equation (1).

3. Upon receiving energy-requesting message, calculates $E_{i, p}$, the energy of next time $\mathrm{p}$ with the equation (2).

4. Reply $E_{i, p}$ to the message sender.

In algorithm 2, a relay station, say $\mathrm{Si}$, saves its energy status of the last three times. It also periodically updates and saves its energy data of the last three times. When $\mathrm{Si}$ receives an energy-requesting message, it calculates $E_{i, p}$, the energy of next time p per the equation (2), and then replies $E_{i, p}$ to the message sender.

Algorithm 3 Find next relay station

1. Insert received energy data to a priority queue.

2. Perform pull() from the priority queue.

3. Use the result of step 2 to figure out the relay station as its next relay station.

In algorithm 3, When a mobile node receives the returned energy data message, its use a priority queue to find the relay with the possible highest energy, and then set the relay station as its next RS to relay the communication.

\section{Performance Evaluation}

We performed the evaluations with a simulated noiseless radio network environment using MATLAB. We create a topology that consists of 20 hexagonal cells. A base station is located in the center of each cell and a relay station is located at each edge. We randomly distribute a number of mobile users to the cells. We performed a sequence of experiments in which the number of mobile users is changed from 200 to 800 with increment of 50 . For each number of mobile users, we measure the aggregate throughput 10 times and present the average. We also measure the average total delay of communications for a large number of pairs of communications.

Our approach considers energy balance with prediction. We compare our approach prediction for energy 
balance("Prediction EB") with an approach without considering energy balance("No EB").

Figure 4 shows that our approach("Prediction EB") results in the less delay. It is because our approach selects the relay station with potential maximum energy as the next RS for communication. Fig. 5 shows our evaluation of aggregate throughput. Of the two approaches, the scheme without energy balance("No EB") has the lower throughput. If one RS runs out of energy and turns down, there will be a hole[13] and the whole throughput will be decreased dramatically. This indicates that energy balance can improve aggregate throughput because the traffic are allocated more reasonable to the relay stations. The throughput of our method is higher.

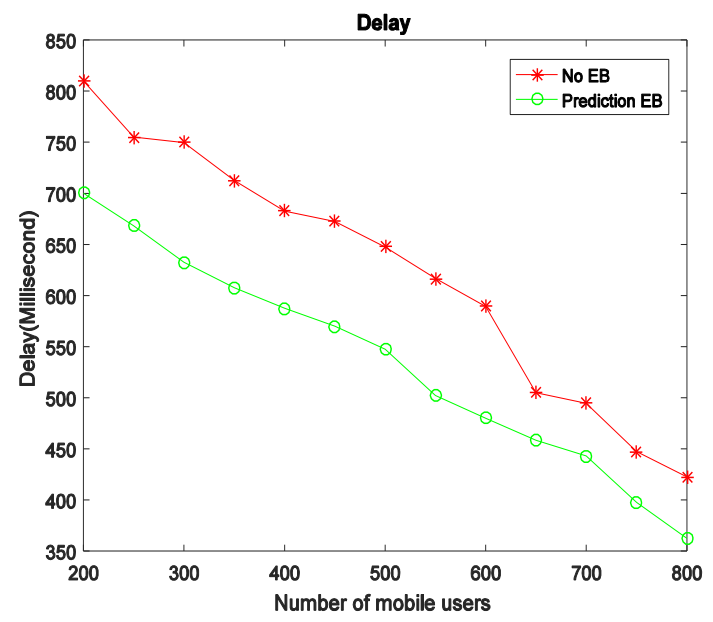

Figure 4. The delay of two approaches

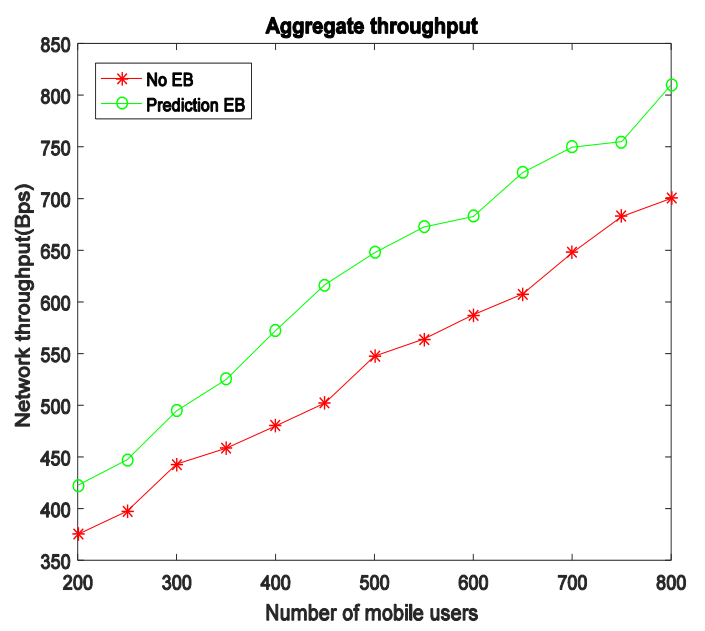

Figure 5. The aggregate thrughtput of two approaches

\section{Conclusion}

In this paper, we present a prediction based mechanism to balance energy utilization in cellular network. In the approach, each relay station only needs to remain the last three status of its energy changes, and then it is able to predict its future energy. The message initiator selects the relay station with potential highest energy to relay data transmission. Simulations demonstrate that our approach results in higher throughput in the network and lower delay over related approaches.

\section{References}

[1] H. Ziaul, H. Boostanimehr and V.K. Bhargava, "Green cellular networks: A survey, some research issues and challenges," Communications Surveys \& Tutorials, IEEE 13.4 (2011 ): 524-540.J. Clerk Maxwell, A Treatise on Electricity and Magnetism, 3rd ed., vol. 2. Oxford: Clarendon, 1892, pp.68-73.

[2] M. Gruber, et al., "EARTH: energy aware radio and network technologies,"Personal, Indoor and Mobile Radio Communications, 2009 IEEE 20th International Symposium on. IEEE, 2009.K. Elissa,

[3] Y. Chen, et al., "Fundamental trade-offs on green wireless net-works,"Communications Magazine, IEEE 49.6 (2011): 30-37.

[4] J. Yang, B. Payne, M. Hitz, Z. Fei, L. Li and T. Wei, "Location Aided Energy Balancing Strategy in Green Cellular Networks," 23th IEEE International Conference on Computer Communication Networks(ICCCN), Workshop on Wireless Mesh and Ad-hoc Networking (WiMAN 2014), Shanghai, China, 2014.

[5] S. Patra, et al., "Improved genetic algorithm for channel allocation with channel borrowing in mobile computing," Mobile Computing, IEEE Transactions on 5.7 (2006): 884-892.

[6] S. Hanly, "An algorithm for combined cell-site selection and power control to maximize cellular spread spectrum capacity," Selected Areas in Communications, IEEE Journal on 13.7 (1995): 1332-1340.

[7] M. Soleimanipour, W. Zhuang and G.H. Freeman, "Optimal resource management in wireless multimedia wideband CDMA systems," SMobile Computing, IEEE Transactions on 1.2 (2002): 143-160.

[8] E. Yanmaz, and O. Tonguz, "Dynamic load balancing and sharing performance of integrated wireless networks," Selected Areas in Communications, IEEE Journal on 22.5 (2004): 862-872.

[9] J. Yang and Z. Fei, "Bipartite Graph Based Dynamic Spectrum Allocation for Wireless Mesh Networks," Distributed Computing Systems Workshops, 2008. ICDCS'08. 28th International Conference on (pp. 96101). IEEE, Beijing, 2008.

[10] X. wang, et al., "A survey of green mobile networks: Opportunities and challenges," Mobile Networks and Applications 17.1 (2012): 4-20.

[11] M. Etoh, T. Ohya and Y. Nakayama, "Energy consumption issues on mobile network systems," 
Applications and the Internet, 2008. SAINT 2008. International Symposium on. IEEE, 2008.

[12] S. Alam, et al., "Energy efficient relay-assisted cellular network model using base station Switching," Globecom Workshops (GC Wkshps), 2012 IEEE. IEEE, 2012.

[13] J. Yang, and Z. Fei, "ITGR: Intermediate Target Based Geographic Routing," Computer Communications and Networks (ICCCN), 2010 Pro-ceedings of 19th International Conference on (pp. 1-6). IEEE, Zurich, Switzerland, August 2010.

[14] J. Yang and Z. Fei, "HDAR: Hole detection and adaptive geographic routing for ad hoc networks," Computer Communications and Networks (ICCCN), Proceedings of 19 th International Conference. IEEE, 2010.

[15] G. Josh, "On Relay-assisted Cellular Networks," thesis, Department of Electrical Engineering, Indian Institute of Technology-Mombay, June, 2010

[16] J. Yang and Z. Fei, "Broadcasting with Prediction and Selective Forward-ing in Vehicular Networks," International Journal of Distributed Sensor Networks, 2013 (2013). 